\title{
ACTIVE METHODS OF INCREASING RELIABILITY OF FBW CONTROL SYSTEMS FOR SMALL AIRCRAFT
}

\section{B. Dolega}

Rzeszow University of Technology, Poland.E-mail: dolbog@prz.rzeszow.pl

Received 1412 2004, accepted 17032005

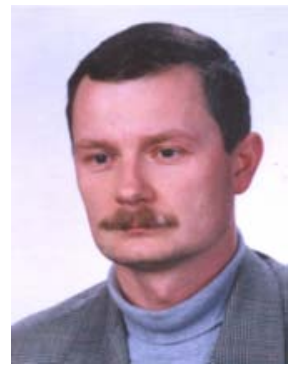

\author{
Boguslaw DOLEGA, $\mathrm{PhD}$ \\ Date and place of birth: 1962 in Jaslo, Poland. \\ Education: Rzeszow University of Technology. \\ Affiliations and functions: Rzeszow University of Technology, assistant; 1994 - PhD degree from the Department of \\ Mechanical Engineering of Rzeszow University of Technology; since 1995 - Professor Assistant on Avionics and \\ Control Systems Department at that University. \\ Research interests: Diagnostics Aircraft Navigation and Control Systems, with a Focus on the Development of the \\ Control Theory for Online Fault Detection and Localization Methods. \\ Experience: Co-organizer of Avionics Conferences. \\ Publications: over 40 scientific articles. \\ Present position: Professor Assistant on Avionics and Control Systems Department at that University.
}

\begin{abstract}
The main task presented in this paper is connected with increasing the reliability of fly-by-wire control systems. The control systems feature a high degree of automatization and employ an intuitive, human-centered way of piloting, makings the aircraft pilot-friendly. But the most important feature of these control systems is safety. Increasing reliability is strongly connected with growth in safety. The high degree of automatization enables the use of different kinds of reconfiguration as an active method of increasing reliability. In this paper, special attention is paid to methods of fault detection and isolation, especially in sensors and actuators connected to the fly-by-wire system. These methods are necessary to build a fault tolerant control system.
\end{abstract}

Keywords: aircraft control system, reconfiguration, fault detection and isolation.

\section{Introduction}

In recent years, air transportation using small aircraft has become very attractive. The number of small aircraft in the U.S.A. will begin increasing by 10,000 per year from 2010 and by 20,000 per year in 2020 [10]. Light planes need not require extensive aviation training. For these aircraft, piloting techniques should be simple and pleasant. Pilots flying in light aircrafts are often not the most highly qualified. These aircraft must therefore be tolerant to the mistakes of pilots. A statistical analysis of accidents clearly shows a significant increase in flight safety. Transportation by small aircraft is cost-effective, but those aircraft aiming at success must offer good quality characteristics in addition to extremely high safety standards. Among other things, these requirements could be satisfied by special avionics systems [7]. Cockpits of modern general aviation aircraft have become similar to cockpits of modern airliners. They have not only flat glass panels but also specific sensors with specialized interfaces. Modern avionics systems in these aircraft can be connect by avionic data buses (ARINC, CAN AEROSPACE, etc.) or standard digital data buses (RS488 , RS-232 etc.). They can integrate avionic system and can be useful in different navigation and control tasks.

In the Avionics and Control System Department of Rzeszow University of Technology research on control and navigation systems for general aviation has been done [12]. One of the conclusions was that the fly-by-wire control systems could guarantee the proper piloting characteristics for these planes. Moreover, these systems could be built in a way that guarantees the reliability necessary for high safety. Not only passive methods, such as using special components with special requirements, but also many active methods increase this reliability. In this paper, active methods of increasing the reliability of the fly-by-wire control systems will be shown. In part I the basic assumption and the basic properties of this system will be presented. The second part includes the description of realization of the experimental fly-by-wire control system that was taken in Rzeszow University of Technology. The next part is connected with specific realizations of some parts of these systems.

\section{Active methods of increasing reliability - basic assumption and some properties}

Control system is a very important in flying safety. The effects of their failures are catastrophic and must be shown to reach the probability of less than $10^{-6}$ per flight hour for the small aircraft to less then $10^{-9}$ per flight hour for the commuters [8]. The designer draws his attention to the flight - while aircraft is airborne. The faults appearing during this time cannot interrupt its mission. Exceptionally it is permissible to end the flight by the safe 
landing. Any repairs of faulty parts during flight are not possible. The consequences of a fault during flight must be clearly indicated to the pilot - both in the case of a simple loss of redundancy and appearing of catastrophic failures.

Computers control all avionics systems included in fly-by-wire system, so designated criteria according to these is quite clear:

- Redundant system architecture with counteracting of the faults appearing,

- Faults and consequences must be apparent to the pilot,

- Faulty component easily identifiable on ground,

- Interoperability - real-time communication of those signals that are necessary to the control process.

Presented conditions are helpful in generating of control action that has a low dependency on the presence of certain faults. Unfortunately, it is not always possible. Therefore during research it was assumed that controlling of the aircraft would be realized with the usage of three levels:

Level I - normal control, all properties of indirect flight control system are employed,

- $\quad$ Level II - simplified control, only the simple CAS (Control Augmentation System) or forming filters are used,

- $\quad$ Level III - emergency control, displacement of aerodynamic control surfaces depends directly on side-stick displacement.

The change of the method is accomplished automatically by a supervisory subsystem. We also consider a manual switching to Level II in the case pilot wishes. Level III should be used only in emergency. These assumptions were very important during the designing process. Some of technical solutions putting into practice directly come from using these levels of control.

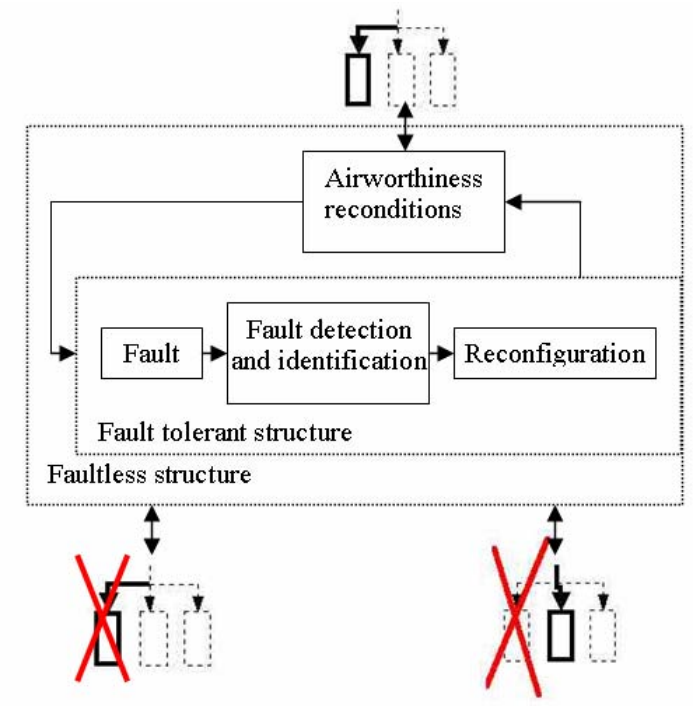

Fig 1. Functional schema of fault tolerant concept
Highly reliable systems use redundancy to achieve fault tolerance due to limited reliability of components or subsystems. Redundancy can be achieved by various means. The straightforward way is multiplication of airborne equipment, but it can also be accomplished using different components that have identical functions Fig 1.

In hardware redundancy, multiple pieces of equipment are used for cross working, and, it is therefore very expensive. Analytic redundancy uses the mathematical model of the system and it is a costeffective method. The analytic redundancy is useful in substitution of hardware. Redundancy is also helpful in diagnosis, especially in fault detection and isolation systems (FDI). The FDI unit provides information about the presence of faults and initiates counteraction. We know that it is very important to recognize that any faulttolerance effect via a control action, regardless of the control strategy applied, is achieved through management of redundancy in the controlled process, such as use of secondary effectors, measurements, transmission buses, etc. Our research leads up to decentralize architecture of the system [12]. But it operates based on a hierarchical way of taking conclusions. Also we take into consideration, maybe most importantly, the functional redundancy aboard an aircraft. It is included in pilot predisposition - not only during normal operation (Level I and II), but also (especially) in abnormal and emergency situations. Only the pilot is able to survey and manage situations that were not foreseen and therefore not considered by the designer. That assumption brings the attention of designers to cognitive pilot reactions. It is especially important to design a control system that is „transparent" to the crew. In other words it is pilotfriendly.

\section{Fly-by-wire control system for PZL-104 aircraft}

In Department of Avionics and Control, Rzeszow University of Technology, a fly-by-wire control system for a PZL-104 "Koliber" has been designed, built, and tested [12]. The general structure of this system is presented in Fig 2. Redundancy as a way of increasing reliability is applied to three independent flight control computers that control double actuators (pitch, roll yaw, and throttle). The pilot selects a control mode using the double control mode selector panels. The throttle level is chosen by the double throttle level interface. The pilot also controls the aircraft by the double Side-Stick interface. Information about movement of the aircraft is provided by:

- $\quad$ three Attitude and Heading Reference Systems (AHRS) - angular orientation,

- two Air Data Computers (ADC) - aircraft movement in relation to air,

- $\quad$ NAV - GNS-530 (GPS, VOR, ILS, comm) and GPS-35 - navigation information, 


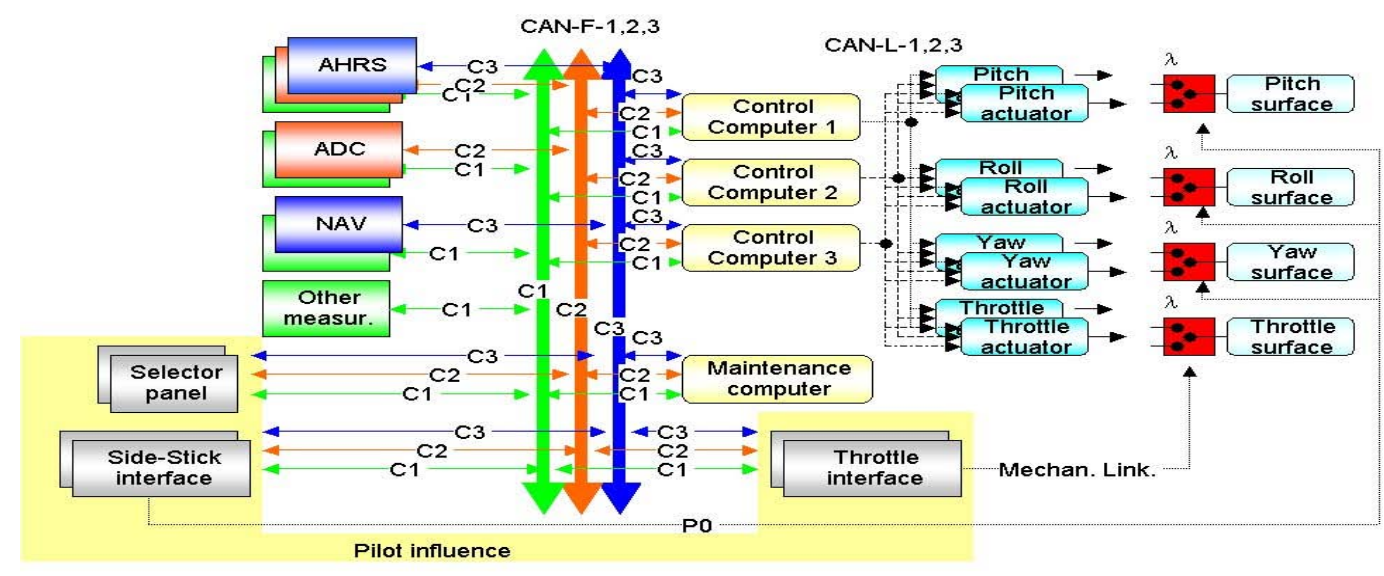

Fig 2. General structure of the fly-by-wire flight control system for PZL-104

Triple digital, high speed, bi-directional data bus network CAN-2 (CAN-F-1, 2, 3) integrates the Control Computers with pilot interfaces and measurement systems. Those systems are connected to the one databus (C1 or $\mathrm{C} 2$ or $\mathrm{C} 3$ ) because that solution is cheaper than others. The actuators are connected to Control Computers through the slow speed version of CAN-2 (CAN-S-1, 2, 3). A lot of data buses and devices connected through these buses led to the adaptation of "CAN aerospace" software as the standard of protocol transmission. This provides more fault tolerant transmission. Each message must contain a status bit field to allow continuous integrity monitoring and minimize failure detection time. Each device connected to the network must inform others about detected failures within itself via a dedicated emergency identifier to support system degradation and maintenance actions. Because the message must contain information about the transmitting station and the type of data associated with the particular message, the network becomes opened. At any time we can connect additional devices (e.g. additional sensors or other measurement systems). Creating a system that is open (open architecture) requires applying software procedures that can identify devices and their signals. The information is necessary to generate vector of efficiency. Control computers generate output signals using that vector (for choice of possible and optimum mode, measure signal etc.). As you see, it is a dynamic process. When a subsystem detects fault in another subsystem, it generates signal that includes that information. It is used by the faulty subsystem for self-reconfiguration and by the others subsystems that cooperate with suspected subsystem.

A PWM (Pulse Duration Modulation) signal standard has been used to directly control the actuators (line P0 - Fig 2.). This action will be taken if two of the three Control Computers malfunction or if the CAN networks that are necessary for proper transmission failed. This level of control will also take place when the absence of the measurement system that is necessary for the mode of flight occurs. The pilot can also switch this level of control as well.
When we talk about the reliability during the flight mod, we ought to note that the aircraft is controlled by four single surfaces. They are not redundant parts. It is easy to prove that the switches $(\lambda$ - Fig 2) that are responsible for the change of the surface control signals must be the most reliable parts. The switch fulfils arbitration between output signals from control computers. The PWM signal (line P0 - Fig 2) has the highest priority.

As was shown, the active methods of increasing reliability are based on information generated by FDI systems. In a fly-by-wire control system, different methods are used to make FDI. In the next paragraph, we try to show some of these methods.

\section{Fault detection and isolation methods}

Fault appearance in a fly-by-wire control system should be fast and reliable detected and isolated. These conditions are important for the proper functioning of the control system during the presence of faults. Faults can appear in different parts and can cause different results. The idea of diagnostic methods is based on the verification of fault symptoms. Each kind of fault has special symptoms for itself and can be diagnosed by the special method for itself. Because we want to make control system more reliable, we are most interested in counteracting the faults that disturb the functioning of the control system. Our fault detection methods should work fast. Diagnostic systems cannot decrease the reliability of diagnosed systems. Therefore they should not be complicated but must provide the most reliable diagnostic information. We use the concept of a dispersed system. Each subsystem provides self-verified information about its diagnostic state. This diagnostic information is taken into consideration by other subsystems as the first diagnostic information. This information is unquestionable and each subsystem turns down cooperation with subsystems that generate information about their failures. Respecting this diagnostic information is very simple and makes system structure very reliable. A lot of subsystems generate this information using simple fault detection methods. They compare some signals with the parameters of its model 
(e.g. limits values, limits values of signal gradients, etc.) [6]. Methods of signal analysis based on the Fourier transformation, calculating the noise value etc. are more complicated. They are used in measurement and navigation systems for initial verification of correct generating of output signals. Using the methods is simple in implementation, not expensive, and rather fast, but diagnostic information is not complete. It is not sensitive to some faults (e.g. some constant value errors). The other detection process is based on the comparison of the real situation with its model. This process will be clearer when we use a more precise model. Using the relationships among some signals of the diagnosed system makes this process clearer. Of course, the other problem is to prepare the proper model itself. Using redundancy can solve this problem. Some redundant parts can be compared to the diagnostic signals generated. We can divide them into hardware and analytic methods. As presented on Fig 2, control computers use redundant measurement and transmission systems. Also, the arbitration systems in actuators make decisions based on redundant signals from control computers (and direct pilot control signals). These systems use e voting methods. By comparing the signals, we decide which of these are improper. Figure 3 shows the generation of the output signal by the arbiter system. We can also see the fault detection process - one of the input signals crossing the lower boundary limit.

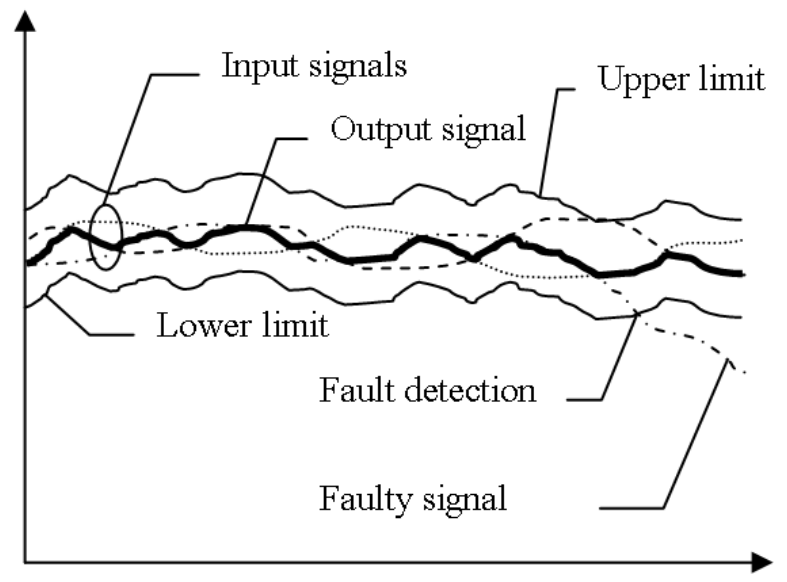

time $\lceil\mathrm{s}\rceil$

Fig 3. Voting system in arbiter

This method is more useful when a single fault appears. It works fast but is not cheap.

If the control system consists of a few microprocessor subsystems, the fault detection methods based on analytic redundancy are cheaper. These methods can be divided in different ways. For example, they could be divided from the point of view of the kind of the model used. Directly, by using the model and the knowledge of its input signals we can generate output signals. Through the comparison of real output signals generated by the model, we make a decision about fault detection. For well-modeled subsystems, which have time invariable characteristics with low uncertainty, this method could be proper for fault detection tasks. In our system we have used this method to diagnose computer systems [8]. In each microprocessor control system, we can find the constant points in algorithm for each work condition. It is, of course, a dynamic system, but complexity depends on quantity states appointed during constructing the algorithm model. It could be easy or complex and depends on the process of designing the diagnostics system. The knowledge of the diagnosed process and its structure is used in a direct way during the synthesis of the fault localization system [6]. The description of flight control system with diagnostic subsystems has been prepared and optimization to receive the maximum diagnostic information with minimum components used has been made. Most diagnosed systems can be modeled with some degrees of accuracy. The assumption of the degree of accuracy is one of the major reasons of choice of the used method of detection. For dynamic subsystems with short time response on input signals, we have implemented a fault detection method based on signal estimation [4]. Of course these methods can be divided in view of linearity, noise, and uncertainty. For example, in Attitude and Heading Reference Systems (AHRS), the models dependent on angular velocities and Euler angles are nonlinear and sensors generate signals with errors (noise) that ought to be taken into consideration. The Kalman filter structure was used in designing the fault detector to this system [1]. In other systems like an Airdata Computer or during fault detection in some actuators, we used diagnostic systems based on the structure of generalized observers [2-4, 9] (linear or nonlinear).

The aircraft thrust control subsystem can be diagnosed by using the model identification method, because fault free and faulty aircraft engine can be describe by models with different parameters [5]. This detection method is being modified and analyzed to be applied in our control system. In all presented methods of analytic redundancy the knowledge of the model of the diagnosed parts is necessary. During the last few years, we noticed interest in using quality and artificial intelligence methods in the fault detection designing process. Special attention should be paid to applying in this task the fuzzy sets and neural networks theory. These methods are especially interesting in the case of absence of the precise description of the diagnosed process. However we need the rough model of this process to make the fuzzy model or data for neuron networks learning.

During the fault localization process, we use the modified diagnostic table method. The fault localization process could be identified to make diagnostic decisions according to the rule - IF \{some conditions\} THEN \{diagnostic localization decision\}[4]. As in most of the systems, which include a human operator, the basic troubles are connected with knowledge representation and the tools used to analyze these systems. Using a result of fault detection's: "R" and modes of flight: "U, $\mathbf{Y}$ " the fault localisation can be obtained on the basis of actual diagnostic knowledge: "D". This means that localisation is the dynamic process with the set of conditions $\mathbf{W}(\mathbf{t})=$ $\{\mathbf{R}(\mathbf{t}), \mathbf{U}(\mathbf{t}), \mathbf{Y}(\mathbf{t}), \mathbf{D}(\mathbf{t})\}$ and the set of decisions $\mathbf{D}=\{\mathbf{D}$ 
$(\mathbf{t}+\mathbf{1}\}$. These signals create the system that can be presented in diagnostic table form. Each row of this table corresponds to some diagnostic statistics. Each column represents different signals from set of attributes WUD. Knowledge is represented by the value of attributes, and the important problem is to find and express relationships among attributes [5]. To make this analysis possible, each signal must take a value from finite sets of values. The coding of real signals from the set of conditions $\mathbf{W}(\mathbf{t})$ to its finite sets of values is the additional problem presented in [4]. Having this description for some faults and diagnostic methods, we are able to analyse the set of tests for each fault. We can make the classification of all states into equivalence classes [11]. Each class presents sets of faults that are not distinguished. We can also analyze the influence of the particular parameters on isolability, sensitivity, and robustness. Finding the relative redacts of this system we can minimise the number of diagnostic subsystems that are necessary to generate the proper fault localisation decision. We can divide our system of detection to parts that we should activate depending on the actual mode of flight and diagnostic state. We have presented the fault detection and localisation system that is the dynamic system. Easy analysis and its modification are the most important features of our system. It can be used in a system of open architecture cooperating with human operator.

\section{Conclusions}

The high reliability of aircraft flight can be achieved by special control architecture that can react on the appearance. As shown, the different kinds of fault detection ought to be used in different parts of the system for faults, which can appear. Using the described method of designing of the fault localization system makes it optimized. This system provides maximum diagnostic information using minimum diagnostic subsystems. Our fault detection and localization system will be proper for the system in which it is built.

\section{Acknowledgements}

This paper was prepared within the framework of the project "Fly-by-wire control system for general aviation aircraft" financed by The Polish State Committee for Scientific Research in the years 2003-2005.

\section{References}

1. Basseville M., Nikiforov I. G. Statistical Change Detection. - Prentice Hall, 1994.

2. Chen J., Patton R.J. Robust Model-based Fault Diagnosis for Dynamic System. - Boston: Kluwer Academic Publishers, 1999.

3. Clark R.N. State Estimation Schemes for Instrument Fault Detection, Fault Diagnosis in Dynamic Systems // Theory and Application / Edited by Patton R.J., Frank P.M. and Clark R.N. - NJ: Prentice Hall, 1989.

4. Dołęga B. Metodyka wykrywania uszkodzeń układów mechanicznych samolotów w trakcie eksploatacji: Rozprawa doktorska. - Rzeszów, 1994.

5. Dołega B. Some concept of diagnostic reasoning for systems with uncertainty $/ / 5^{\text {th }}$ International Multidisciplinary Conference, Baiia Mare 2001. 2001.

6. Dołęga B. Wybrane aspekty rozwiązań programowych zastosowane w autopilocie dla samolotów lokalnej komunikacji // Prace Instytutu Lotnictwa. - 1993. - Vol 134, No 3. - P. 47-61.

7. Dołega B., Rogalski T. The method of aircraft control system graduating // 6th International Seminar on Recent Research and design progress in aeronautical engineering and its influence on education. - Riga, 2004.

8. Flight Control Design - Best Practices, RTO-TR-29, NATO. -2000.

9. Gertler J.J. Fault Detection and Diagnosis in Engineering Systems. - New York: Marcel Dekker, 1998.

10. Holmes B.J. AGATE Overview // World Aviation Conference, Anaheim CA, 1998. - 1998.

11. Pawlak Z. Rough Sets. Theoretical Aspects of Reasoning about Data. - Kluwer Academic Publishers, 1991.

12. Tomczyk A. Facilitated Airplane - project and preliminary in-flight experiments // Aerospace Science and Technology. - Elsevier, 2004. - Vol 8. - P. 469-477. 